\title{
miR-224 overexpression is a strong and independent prognosticator of short-term relapse and poor overall survival in colorectal adenocarcinoma
}

\author{
PANAGIOTIS G. ADAMOPOULOS ${ }^{1}$, CHRISTOS K. KONTOS ${ }^{1}$, STAMATIA-MARIA RAPTI ${ }^{1}$, \\ IORDANIS N. PAPADOPOULOS ${ }^{2}$ and ANDREAS SCORILAS ${ }^{1}$ \\ ${ }^{1}$ Department of Biochemistry and Molecular Biology, University of Athens, Panepistimiopolis; \\ ${ }^{2}$ Fourth Surgery Department, University of Athens, University General Hospital 'Attikon', Athens, Greece
}

Received October 7, 2013; Accepted December 4, 2013

DOI: 10.3892/ijo.2014.2775

\begin{abstract}
Colorectal adenocarcinoma constitutes the most frequent form of colorectal cancer and a serious cause of cancer-related deaths. The expression of multiple miRNAs, including miR-224, is deregulated in colorectal adenocarcinoma. The aim of this study was the investigation of the prognostic value of miR-224 in colorectal adenocarcinoma. For this purpose, total RNA was isolated from 115 colorectal adenocarcinomas and 66 adjacent non-cancer mucosae. Total RNA $(2 \mu \mathrm{g})$ was polyadenylated and reverse transcribed. A quantitative PCR method based on SYBR-Green chemistry was developed and applied for the quantification of miR-224 levels, followed by extensive biostatistical analysis. miR-224 levels in malignant colorectal adenocarcinomas ranged between 1.81 and $187.75 \mathrm{RQU}$ (miR-224 copies/1,000 SNORD48 copies) with a median of 34.27 , and were significantly elevated, compared to miR-224 levels in adjacent non-cancer mucosae $(\mathrm{p}<0.001)$. Enhanced miR-224 expression constitutes a rather strong prognosticator in colorectal adenocarcinoma, predicting short-term relapse and poor overall survival in these patients $(\mathrm{p}=0.012$ and $p=0.005$, respectively), independent of established clinicopathological parameters. In conclusion, miR-224 is significantly upregulated in malignant colorectal tumors compared to adjacent non-cancer mucosae, and its enhanced expression constitutes an independent predictor of short-term relapse and poor overall survival in colorectal adenocarcinoma patients.
\end{abstract}

\section{Introduction}

MicroRNAs (miRNAs) constitute a class of small non-coding RNAs of approximately 19-25 nucleotides that post-transcrip-

Correspondence to: $\mathrm{Dr}$ Andreas Scorilas, Department of Biochemistry and Molecular Biology, University of Athens, Panepistimiopolis, Athens 15701, Greece

E-mail: ascorilas@biol.uoa.gr

Key words: microRNAs, microRNA-224, colorectal cancer, prognostic tumor biomarker, real-time PCR tionally regulate gene expression. These small RNA molecules regulate or tune finely protein-coding gene expression in animals and plants. miRNAs usually bind to the 3'-untranslated region (3'-UTR) of target mRNAs, leading to mRNA degradation and/or translational repression (1). Therefore, they play significant roles in cell proliferation, cell death, as well as many other important cellular processes $(2,3)$. Consequently, miRNAs have emerged as significant molecules that are implicated in carcinogenesis and metastasis of many different cancer cell types. miRNAs in carcinogenesis can target transcripts of tumor-suppressor genes and/or proto-oncogenes.

Colorectal cancer (CRC) is one of the most frequent cancers and a serious cause of cancer-related deaths all over the world (4). About 5-10\% of all colorectal cancers develop as a defined hereditary cancer syndrome. The two main forms of hereditary cancer syndromes are hereditary non-polyposis colorectal cancer (HNPCC) and familial adenomatous polyposis (FAP) (5). Recent studies have pointed out the connection between CRC and the expression levels of specific miRNAs such as miR-143 and miR-145, in an attempt to clarify their role in carcinogenesis in colonic mucosae (6).

miR-224 expression is deregulated in several human malignancies, including prostate $(7,8)$, breast $(9)$, ovarian (10) and cervical cancer (11), pancreatic ductal adenocarcinoma (12), hepatocellular carcinoma (13-15), colorectal cancer (16-18), thyroid cancer (19), glioma (20), as well as acute myeloid leukemia (21), strongly suggesting that miR-224 expression plays a role in the general process of carcinogenesis. With regard to prostate cancer, miR-224 is expressed by perineural cancer cells and is likely to be involved in perineural invasion, which constitutes the dominant pathway for local invasion in this cancer (7). Overexpression of miR-224 plays a major role in Hep G2 cells, as it is considered to regulate the migration and invasion of these hepatocellular carcinoma cells (15). Moreover, high miR-224 levels in highly invasive and metastatic pancreatic ductal adenocarcinoma account for downregulation of CD40 protein expression at the cell surface (12).

miR-224 targets protein-coding genes, modulates the levels of the respective proteins and is thus involved in the pathogenesis of several diseases. The apoptosis inhibitor 5 
(API5) (13), whose expression prevents apoptosis after growth factor deprivation (22) is a target of miR-224. By downregulating API5 expression, miR-224 increases apoptotic cell death of Hep G2 cells; however, miR-224 overexpression in these cells was also shown to increase cell proliferation (13). API5 depletion sensitizes cancer cells to chemotherapy (22). Significantly augmented API5 expression has been detected in biopsies of lung and colorectal tumors, compared to biopsies from adjacent normal tissues. Therefore, API5 protein is upregulated in tumor epithelial cells and has been proposed as a potential prognostic marker in colorectal cancer (23).

Furthermore, in breast cancer cells miR-224 compromises the expression of RAF1 kinase inhibitor protein (RKIP), a tumor suppressor that protects against metastasis and genomic instability (9). A recent study showed that RKIP expression is diffused in normal colorectal mucosa and progressively lost towards tumor center and front. Loss of RKIP expression predicts features of epithelial-mesenchymal transition (EMT) and is also associated with distant metastasis; however, its prognostic significance is restricted to the tumor center (24). The CD40 gene constitutes another confirmed target of miR-224 (12). CD40, a member of the tumor necrosis factor receptor (TNFR) superfamily, is a co-stimulatory protein found on antigen presenting cells and is required for their activation (25). CD40 is highly expressed in various established human CRC cell lines in culture as well as in CRC specimens. Activation of this receptor by membrane-bound CD40L, but not by soluble agonists, has been shown to trigger programmed cell death in CD40-positive CRC cells and to induce secretion of proinflammatory cytokines (26).

The aim of this study was to investigate the prognostic potential of miR-224 expression and its putative clinical application in colorectal adenocarcinoma prognosis. We developed a highly sensitive quantitative real-time PCR methodology and used it to quantify miR-224 levels in malignant colorectal tumors and adjacent non-cancerous colorectal mucosae.

\section{Materials and methods}

Tissue samples. We collected 115 cancerous and 66 paired non-cancerous colorectal tissue specimens from patients who underwent surgical treatment for primary colorectal adenocarcinoma at the University General Hospital 'Attikon', between 2000 and 2010. Tumor tissues were histologically characterized by a pathologist and frozen in liquid nitrogen immediately after resection. Informed consent was obtained from all patients participating in the study. The study was approved by the institutional Ethics Committee of the University General Hospital 'Attikon' (Athens, Greece) and performed in accordance with the ethical standards of the 1964 Declaration of Helsinki and its later amendments.

Patients' clinicopathological parameters included the tumor size, histological grade and stage of the disease according to the TNM classification. The TNM staging system updated in May, 2011 (27), combines tumor invasion $(\mathrm{T})$, regional lymph node status $(\mathrm{N})$, and presence or absence of distant metastases (M). Patients' clinical and biologic characteristics are summarized in Table I.
Follow-up information included disease status (diseasefree or recurrence) and survival status (alive or deceased), along with the dates of the events and the cause of death. The median disease-free survival (DFS) was 32.0 months (range, 3.0-120.0) and the median overall survival (OS) was 31.0 months (range, 1.0-120.0). Patient age ranged from 37.0 to 93.0 years with a mean $( \pm \mathrm{SE})$ of $67.8( \pm 1.1)$ (Table II).

Human CRC cell line culture. Human colorectal adenocarcinoma Caco-2 cells were subcultured in DMEM medium, adjusted to contain $10 \%$ fetal bovine serum (FBS), $100 \mathrm{kU} / 1$ penicillin, $0.1 \mathrm{~g} / 1$ streptomycin and $2 \mathrm{mM}$ L-glutamine. Cells were seeded at a concentration of $0.5 \times 10^{5}$ cells $/ \mathrm{ml}$ and incubated for $48 \mathrm{~h}$ at $37^{\circ} \mathrm{C}$, in a humidified atmosphere containing $5 \% \mathrm{CO}_{2}$, before being collected for further use.

Total RNA extraction, polyadenylation and reverse transcription. Tissue specimens were pulverized and then dissolved in TRI Reagent ${ }^{\circledR}$ (Molecular Research Center, Inc. Cincinnati, $\mathrm{OH}$, USA). Following the manufacturer's instructions, total RNA was extracted from homogenized tumors and Caco-2 cells, diluted in RNA Storage Solution (Life Technologies Ltd., Carlsbad, CA, USA), and stored at $-80^{\circ} \mathrm{C}$ until use. The concentration and purity of total RNA were assessed spectrophotometrically at 260 and $280 \mathrm{~nm}$. Next, polyadenylation of total RNA and reverse transcription into first-strand cDNA was performed, as previously described (8). In brief, $2 \mu \mathrm{g}$ of total RNA were polyadenylated using recombinant poly(A) polymerase (New England Biolabs Ltd., Whitby, ON, Canada) in the presence of ATP $(80 \mu \mathrm{M})$. Next, first-strand cDNA was synthesized from $2 \mu \mathrm{g}$ of polyadenylated total RNA, using M-MLV Reverse Transcriptase (Life Technologies Ltd.) and an oligo-dT-adapter sequence (5'-GCGAGCACAGAATTAA TACGACTCACTATAGGTTTTTTTTTTTTVN-3', where $\mathrm{V}=\mathrm{G}, \mathrm{A}, \mathrm{C}$ and $\mathrm{N}=\mathrm{G}, \mathrm{A}, \mathrm{T}, \mathrm{C}$ ) as primer. The final reaction volume was $20 \mu$ l.

Quantitative real-time PCR. Quantitative real-time PCR (qPCR) was performed using the SYBR-Green chemistry in a 7500 Fast Real-Time PCR System (Applied Biosystems, Foster City, CA, USA). Based on the published sequences of mature miR-224 and SNORD48 (small nucleolar RNA, C/D box 48; also known as U48 or RNU48) with GenBank accession numbers NR_029638.1 and NR_002745.1, respectively, two miRNA gene-specific primers were designed and used in combination with a common reverse primer to generate two respective amplicons. The sequence of the miR-224 forward primer was 5'-CAAGTCACTAGTGGTTCCGTTAA-3' and that of the SNORD48 forward primer was 5'-TGATGATGAC CCCAGGTAACTCT-3'. The sequence of the common reverse primer, complementary to the oligo-dT-adapter, was 5'-GCGA GCACAGAATTAATACGAC-3'. The resulting PCR amplicons for miR-224 and SNORD48 were 65- and 105-bp long, respectively. The reaction mixture contained $1 \mu \mathrm{l}$ of 10 -fold diluted cDNA, $5 \mu$ l KAPA ${ }^{\mathrm{TM}}$ SYBR $^{\circledR}$ FAST qPCR Kits (2X) (Kapa Biosystems Inc., Woburn, MA, USA), and $2 \mu \mathrm{l}$ of gene-specific primers (final concentration: $200 \mathrm{nM}$ each), in a final reaction volume of $10 \mu \mathrm{l}$. The cycling conditions were as follows: a denaturation step at $95^{\circ} \mathrm{C}$ for $3 \mathrm{~min}$, followed by 40 cycles of $95^{\circ} \mathrm{C}$ for $3 \mathrm{sec}$, for denaturation of the PCR prod- 
Table I. Clinical and biological characteristics of colorectal adenocarcinoma patients.

\begin{tabular}{lc}
\hline Variable & No. of patients $(\%)$ \\
\hline Total & 115 \\
Sex & \\
Male & 60 \\
Female & 55 \\
Histological grade & \\
I & $13(11.3 \%)$ \\
II & $79(68.7 \%)$ \\
III & $23(20.0 \%)$ \\
T & \\
T1 & $2(1.8 \%)$ \\
T2 & $12(10.4 \%)$ \\
T3 & $68(59.1 \%)$ \\
T4 & $33(28.7 \%)$ \\
N & \\
N0 & $67(58.3 \%)$ \\
N1 & $30(26.1 \%)$ \\
N2 & $18(15.6 \%)$ \\
M & \\
M0 & $102(88.7 \%)$ \\
M1 & $13(11.3 \%)$ \\
TNM stage & \\
I & $13(11.3 \%)$ \\
II & $52(45.2 \%)$ \\
III & $37(32.2 \%)$ \\
\hline & $13(11.3 \%)$ \\
\hline
\end{tabular}

T, tumor invasion; $\mathrm{N}$, nodal status; $\mathrm{M}$, metastasis; TNM, tumor, nodal status and metastasis.

ucts, and $60^{\circ} \mathrm{C}$ for $30 \mathrm{sec}$, for primer annealing and extension. Each real-time PCR reaction was performed in duplicate to evaluate the reproducibility of data (Fig. 1A).

In order to distinguish between the specific PCR products and primer-dimers or other non-specific products, melting curves of the PCR products were generated after amplification, by heating the reaction mixtures from 60 to $95^{\circ} \mathrm{C}$ with a heating rate of $0.3^{\circ} \mathrm{C} / \mathrm{sec}$ and continuously acquiring fluorescence emission data. Primer-dimers and/or other non-specific products are characterized by a lower $\mathrm{T}_{\mathrm{m}}$ than those of the miR-224 and SNORD48 amplicons (Fig. 1B).

Calculations and validation of the comparative $C_{T}\left(2^{-\Delta \Delta C T}\right)$ method for miR-224 quantification. Calculations were made using the comparative $\mathrm{C}_{\mathrm{T}}\left(2^{-\Delta \Delta \mathrm{CT}}\right)$ method. The prerequisites for the application of the comparative $C_{T}\left(2^{-\Delta \Delta C T}\right)$ method (28) were checked in a validation experiment, in which $\mathrm{C}_{\mathrm{T}}$ values of miR-224 and SNORD48 were measured in a dilution series of Caco-2 cDNA. Real-time PCR efficiency $(E)$ for amplification of each gene was calculated using the following formula: $E=-1+10^{(-1 / \alpha)}$, where $\alpha$ is the slope of the corresponding amplification plot. As illustrated in Fig. 1C, the slopes of miR-224 and SNORD48 amplification plots are
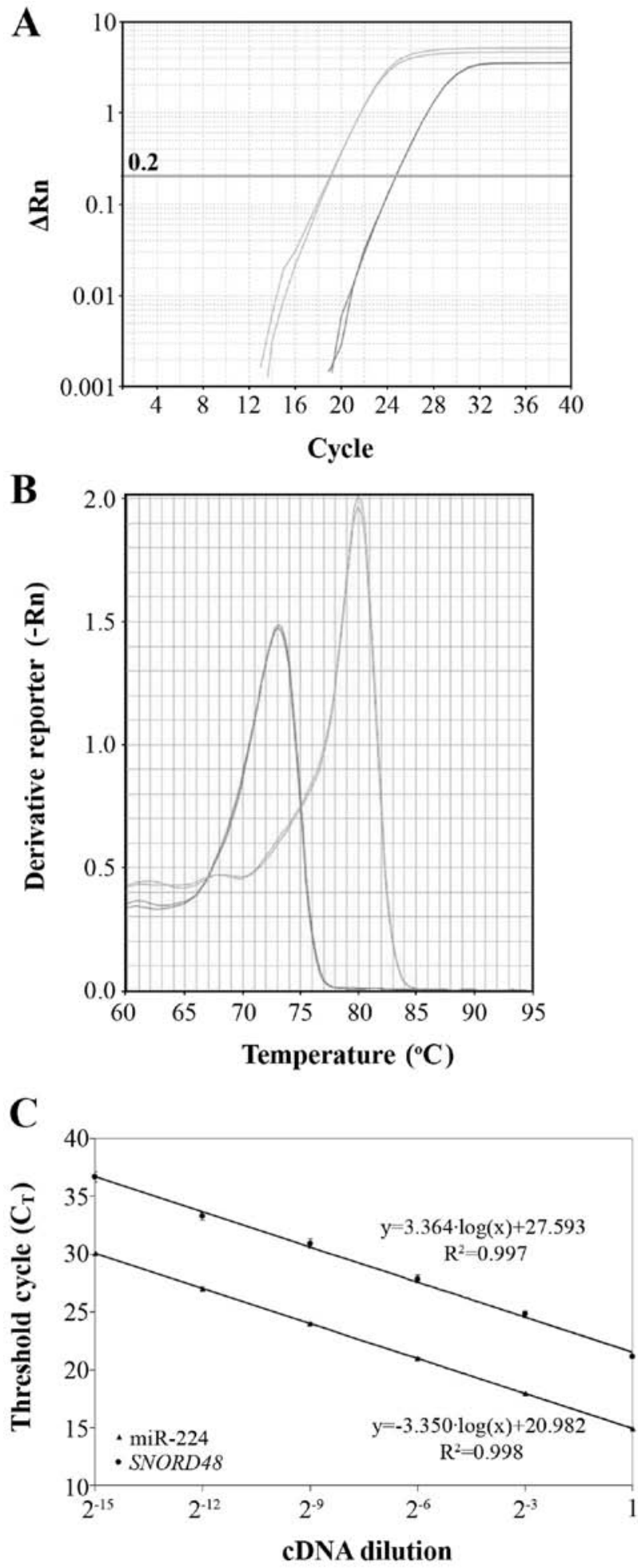

Figure 1. Real-time PCR quantification of miR-224 expression in colorectal adenocarcinoma tissues. (A) Amplification plots of miR-224 and SNORD48, (B) dissociation curves of the respective amplicons, and (C) validation of the comparative $\mathrm{C}_{\mathrm{T}}\left(2^{-\Delta \triangle \mathrm{CT}}\right)$ method, in order to assess the efficiency of amplification of miR-224 and SNORD48.

very similar ( -3.364 and -3.350 , respectively), which clearly indicates similar efficiencies for the corresponding amplicons ( 98.3 and $98.8 \%$, respectively).

In the current study, SNORD48 was used as an endogenous control gene so as to normalize PCRs for the RNA amount 
Table II. Distribution of the numerical variables of the study in the cohort of colorectal adenocarcinoma patients.

\begin{tabular}{|c|c|c|c|c|c|}
\hline \multirow[b]{2}{*}{ Variable } & \multirow[b]{2}{*}{ Mean \pm SE } & \multirow[b]{2}{*}{ Range } & \multicolumn{3}{|c|}{ Percentile } \\
\hline & & & 25 th & $\begin{array}{c}\text { 50th } \\
\text { (median) }\end{array}$ & 75th \\
\hline \multicolumn{6}{|l|}{ miR-224 expression (RQU) } \\
\hline in tumors $(\mathrm{n}=115)$ & $47.39 \pm 4.02$ & $1.81-187.75$ & 16.14 & 34.27 & 64.30 \\
\hline in non-cancerous specimens $(\mathrm{n}=66)$ & $15.84 \pm 1.74$ & $2.16-63.25$ & 6.10 & 11.34 & 21.25 \\
\hline Patient age (years) & $67.8 \pm 1.1$ & $37.0-93.0$ & 59.0 & 69.0 & 77.0 \\
\hline Tumor size $\left(\mathrm{cm}^{2}\right)$ & $23.2 \pm 1.8$ & $0.8-132.0$ & 10.5 & 18.2 & 30.0 \\
\hline DFS (months) & $39.9 \pm 3.2$ & $3.0-120.0$ & 17.0 & 32.0 & 52.0 \\
\hline OS (months) & $40.5 \pm 2.9$ & $1.0-120.0$ & 18.0 & 31.0 & 53.0 \\
\hline
\end{tabular}

SE, standard error of the mean; RQU, relative quantification units; DFS, disease-free survival; OS, overall survival.

added to the reverse transcription reactions, while the colorectal adenocarcinoma cell line Caco-2 was used as a calibrator in order to make PCRs from distinct runs comparable (29).

The normalized result of each sample was calculated as the ratio of miR-224 copies to SNORD48 copies divided by the same ratio that had been previously calculated for Caco- 2 cells. The normalized $\left(2^{-\Delta \Delta C T}\right)$ amounts of tissue sample miR-224 levels were then multiplied with the average ratio of miR-224 copies to SNORD48 copies of Caco-2 cells (2-6.611), calculated by the difference between the $y$-intercepts of the regression lines shown in Fig. 1C. This procedure produced comparable results that do not depend on the miR-224 expression levels of Caco- 2 cells. Finally, normalized results were multiplied by 1,000 and designated as relative quantification units (RQU), standing for miR-224 copies/1,000 SNORD48 copies.

Statistical analysis. As the distribution of the expression levels of miR-224 in our cohort of patients was not Gaussian, analysis of the differences in the two groups of specimens (colorectal adenocarcinomas vs. non-cancerous mucosae) was performed with the non-parametric Mann-Whitney U test. Moreover, miR-224 levels between paired tissue samples were compared using the Wilcoxon signed-rank test.

So as to determine the optimal cut-off point for categorization of patients into miR-224-positive and miR-224-negative as there are no established cut-off points, we used the X-tile software, an algorithm that facilitated the determination of an optimal cut-off point by correcting for the use of minimum p-value statistics (30). This cut-off point was 37.79 RQU, equal to the 56th percentile. According to this cut-off value, miR-224 expression in each specimen was categorized as negative or positive.

We also constructed receiver operating characteristic (ROC) curves for miR-224 expression levels, by plotting sensitivity versus (1-specificity) and the areas under the ROC curves (AUC) were analyzed by Hanley and McNeil method. To further examine the discriminatory value of miR-224 expression in colorectal adenocarcinoma, we performed univariate binary logistic regression analysis.

Associations between miR-224 expression status and survival of the patients were assessed by Kaplan-Meier
DFS and OS curves. The differences between the curves were evaluated by the log-rank (Mantel-Cox) test. We also developed Cox proportional hazard regression models, so as to assess the association between the prognostic markers and the relative risks for relapse and death of patients. Multivariate Cox regression models were adjusted for the aforementioned established clinicopathological parameters. Finally, Kaplan-Meier survival analysis was carried out to evaluate the prognostic potential of miR-224 expression with regard to DFS and OS in groups of patients, stratified according to distinct clinicopathological features. The level of statistical significance was defined at a probability value of less than $0.05(\mathrm{p}<0.05)$.

\section{Results}

miR-224 expression levels in colorectal adenocarcinoma tissue specimens and adjacent non-cancerous colorectal mucosae. miR-224 expression was significantly higher in colorectal adenocarcinoma tissues than in non-cancerous mucosae, ranging from 1.81 to $187.75 \mathrm{RQU}$ with a mean $( \pm \mathrm{SE})$ of $47.39( \pm 4.02)$ in the former, while varying between 2.16 and $63.25 \mathrm{RQU}$ with a mean $( \pm \mathrm{SE})$ of $15.84( \pm 1.74)$ in the latter (Table II and Fig. 2A). Comparison of miR-224 levels between colorectal tumors and their adjacent non-cancerous mucosae uncovered the profound overexpression of this molecule in the malignant colorectal tumors [55 out of $66(83.3 \%)$ examined tissue pairs, p<0.001; Fig. 2B].

miR-224 expression of each colorectal adenocarcinoma was then categorized into one of two groups (positive or negative), as described in Materials and methods; thus, 51 (44.3\%) cases were classified as miR-224-positive and $64(55.7 \%)$ as miR-224-negative.

Discriminatory value of miR-224 expression in colorectal adenocarcinoma. So as to evaluate the potential of miR-224 expression as a predictive biomarker for the discrimination between colorectal adenocarcinoma and non-cancerous colorectal tissues, we performed ROC and logistic regression analyses. As illustrated by the ROC curve in Fig. 3, miR-224 expression was found to distinguish very efficiently 

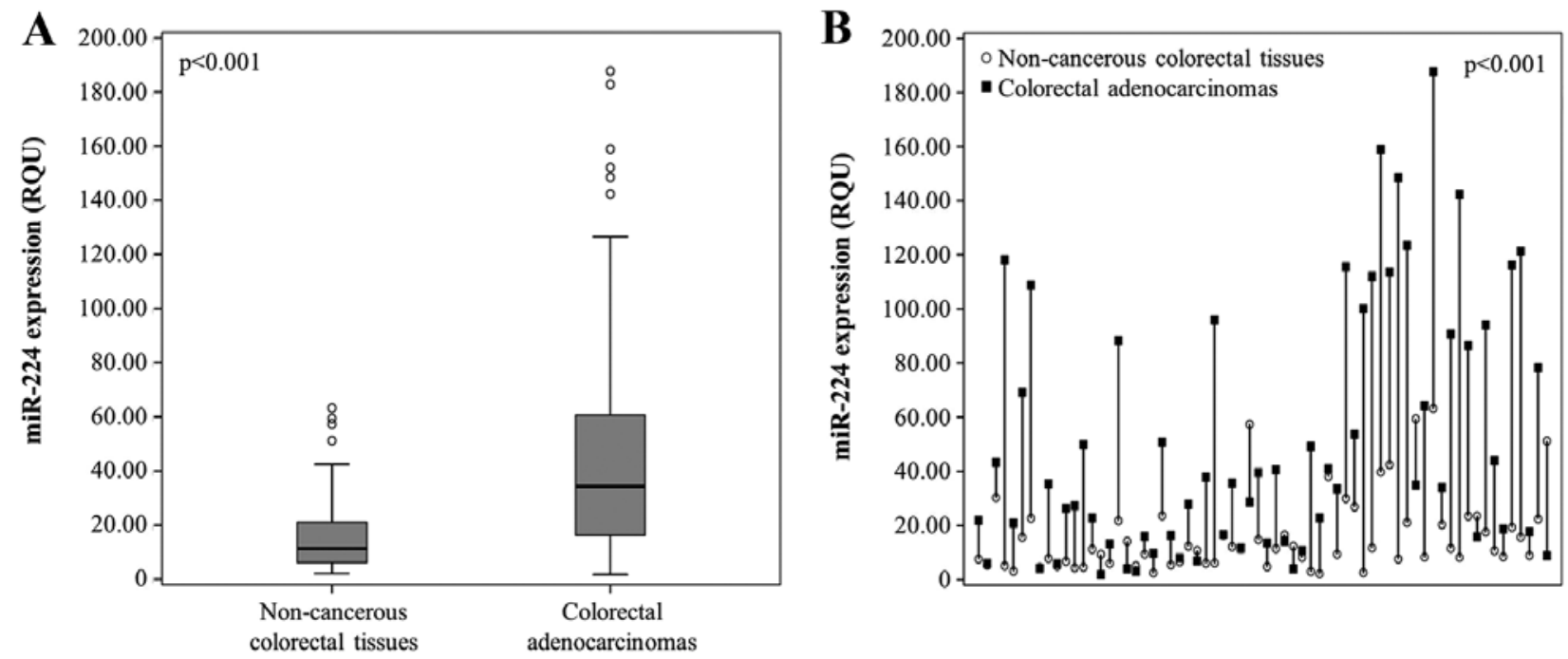

Figure 2. Comparison of the distribution of miR-224 expression in colorectal tumors and non-cancerous tissue specimens. (A) miR-224 transcripts are more abundant in the cohort of colorectal adenocarcinoma specimens. (B) miR-224 expression is significantly upregulated in colorectal adenocarcinoma specimens than in paired adjacent non-malignant tissues.

colorectal adenocarcinoma from healthy colorectal mucosae [area under the curve $(\mathrm{AUC})=0.78,95 \%$ confidence interval $(95 \% \mathrm{CI})=0.71-0.85, \mathrm{p}<0.001]$.

Univariate logistic regression analysis revealed that high miR-224 levels constitute a predictor of the presence of colorectal adenocarcinoma. Analysis of miR-224 expression as a dichotomous variable showed that miR-224 positivity in colorectal mucosae predicts a 7-fold higher risk for adenocarcinoma (crude odds ratio $=6.72,95 \% \mathrm{CI}=2.83-15.96$, $\mathrm{p}<0.001)$.

miR-224 expression predicts short-term relapse in colorectal adenocarcinoma patients. Follow-up information was available for 104 patients; however, 13 patients were diagnosed with distant metastases before or at the time of surgery and were excluded from DFS analysis. Out of the remaining 91 patients, $15(16.5 \%)$ relapsed during the respective follow-up periods. In Cox univariate regression analysis (Table III), a 3.5-fold higher risk of recurrence was predicted for colorectal adenocarcinoma patients bearing tumors with positive miR-224 expression status [hazard ratio $(\mathrm{HR})=3.52,95 \% \mathrm{CI}=1.20-10.33, \mathrm{p}=0.022)$. We also performed Kaplan-Meier survival analysis so as to evaluate miR-224 expression in terms of predicting DFS. In agreement with the aforementioned results, Kaplan-Meier DFS curves illustrated that colorectal adenocarcinoma patients with miR-224-positive tumors had significantly shorter DFS $(\mathrm{p}=0.014)$, compared to those who had a miR-224-negative colorectal adenocarcinoma (Fig. 4A).

In the multivariate Cox regression analysis (Table III), miR-224 expression predicted a significantly unfavorable prognostic outcome $(\mathrm{HR}=4.61,95 \% \mathrm{CI}=1.41-15.09, \mathrm{p}=0.012)$, which was independent of tumor size, histological grade, tumor invasion, and regional lymph node status.

miR-224 expression as an independent prognosticator of poor OS in colorectal adenocarcinoma. Regarding OS, out of 104 colorectal adenocarcinoma patients for whom follow-

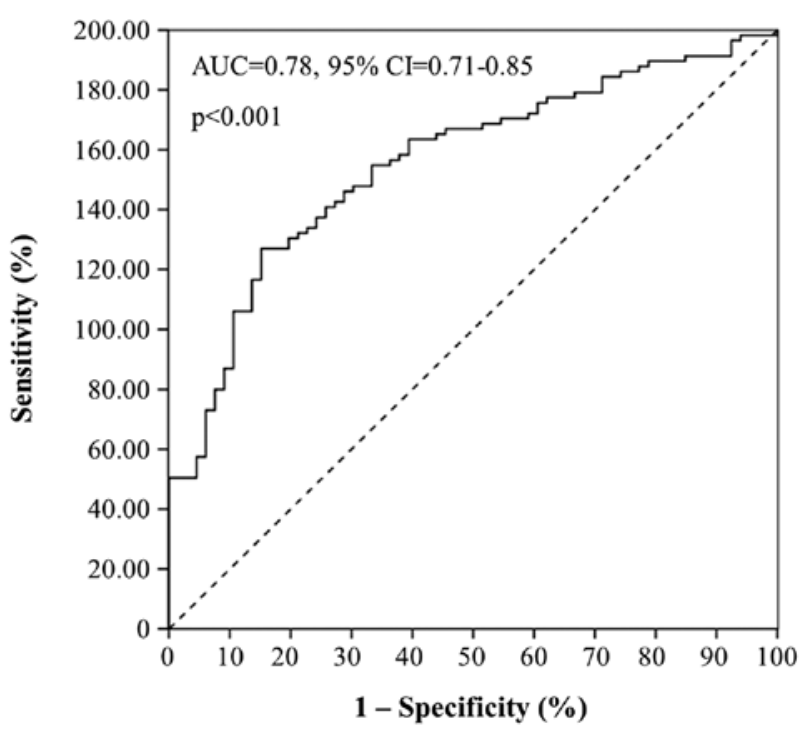

Figure 3. ROC curve for miR-224 expression. miR-224 expression was found to distinguish successfully colorectal adenocarcinoma from non-cancerous colorectal tissues.

up data were available, 24 patients (23.1\%) died during the accrual follow-up period. Cox univariate regression analysis (Table IV) demonstrated that patients with miR-224-positive colorectal adenocarcinoma were at higher risk of death $(\mathrm{HR}=4.08,95 \% \mathrm{CI}=1.68-9.88, \mathrm{p}=0.002)$, compared to patients whose colorectal adenocarcinoma was miR-224-negative. Hence, enhanced miR-224 expression seems also to constitute a strong unfavorable predictor of OS. Histological grade of the tumor as well as TNM stage, which is a resultant of tumor invasion, regional lymph node status and presence of distant metastases, were significant prognosticators of OS $(\mathrm{p}=0.014$ and $\mathrm{p}<0.001$, respectively), as expected. In accordance with these results, Kaplan-Meier OS analysis revealed 
Table III. miR-224 expression and DFS of colorectal adenocarcinoma patients.

\begin{tabular}{|c|c|c|c|c|c|c|}
\hline \multirow[b]{3}{*}{ Covariate } & \multicolumn{6}{|c|}{ Disease-free survival } \\
\hline & \multicolumn{3}{|c|}{ Univariate analysis $(\mathrm{n}=91)$} & \multicolumn{3}{|c|}{ Multivariate analysis $^{\mathrm{d}}(\mathrm{n}=91)$} \\
\hline & $\mathrm{HR}^{\mathrm{b}}$ & $95 \% \mathrm{CI}^{\mathrm{c}}$ & p-value & $\mathrm{HR}^{\mathrm{b}}$ & $95 \% \mathrm{CI}^{\mathrm{c}}$ & p-value \\
\hline \multicolumn{7}{|l|}{ miR-224 expression status ${ }^{\mathrm{a}}$} \\
\hline Negative & 1.00 & & & 1.00 & & \\
\hline Positive & 3.52 & $1.20-10.33$ & 0.022 & 4.61 & $1.41-15.09$ & 0.012 \\
\hline Tumor size & 0.96 & $0.92-1.01$ & 0.10 & 0.93 & $0.87-1.00$ & 0.061 \\
\hline Histological grade (ordinal) & 2.33 & $0.91-5.96$ & 0.078 & 6.30 & $1.61-24.65$ & 0.008 \\
\hline $\mathrm{T}$ (ordinal) & 1.36 & $0.59-3.17$ & 0.47 & 1.74 & $0.72-4.24$ & 0.22 \\
\hline Positive nodal status & 1.01 & $0.48-2.13$ & 0.98 & 0.43 & $0.16-1.15$ & 0.093 \\
\hline
\end{tabular}

HR, hazard ratio; CI, confidence interval; T, tumor invasion; ${ }^{\mathrm{a}}$ cut-off point: $37.79 \mathrm{RQU}$, equal to the 56th percentile; ${ }^{\mathrm{b}}$ estimated from Cox proportional hazard regression model; ${ }^{\mathrm{c}}$ confidence interval of the estimated HR; ${ }^{\mathrm{d}}$ multivariate models were adjusted for tumor size, histological grade, tumor invasion $(\mathrm{T})$, and patients' nodal status $(\mathrm{N})$.
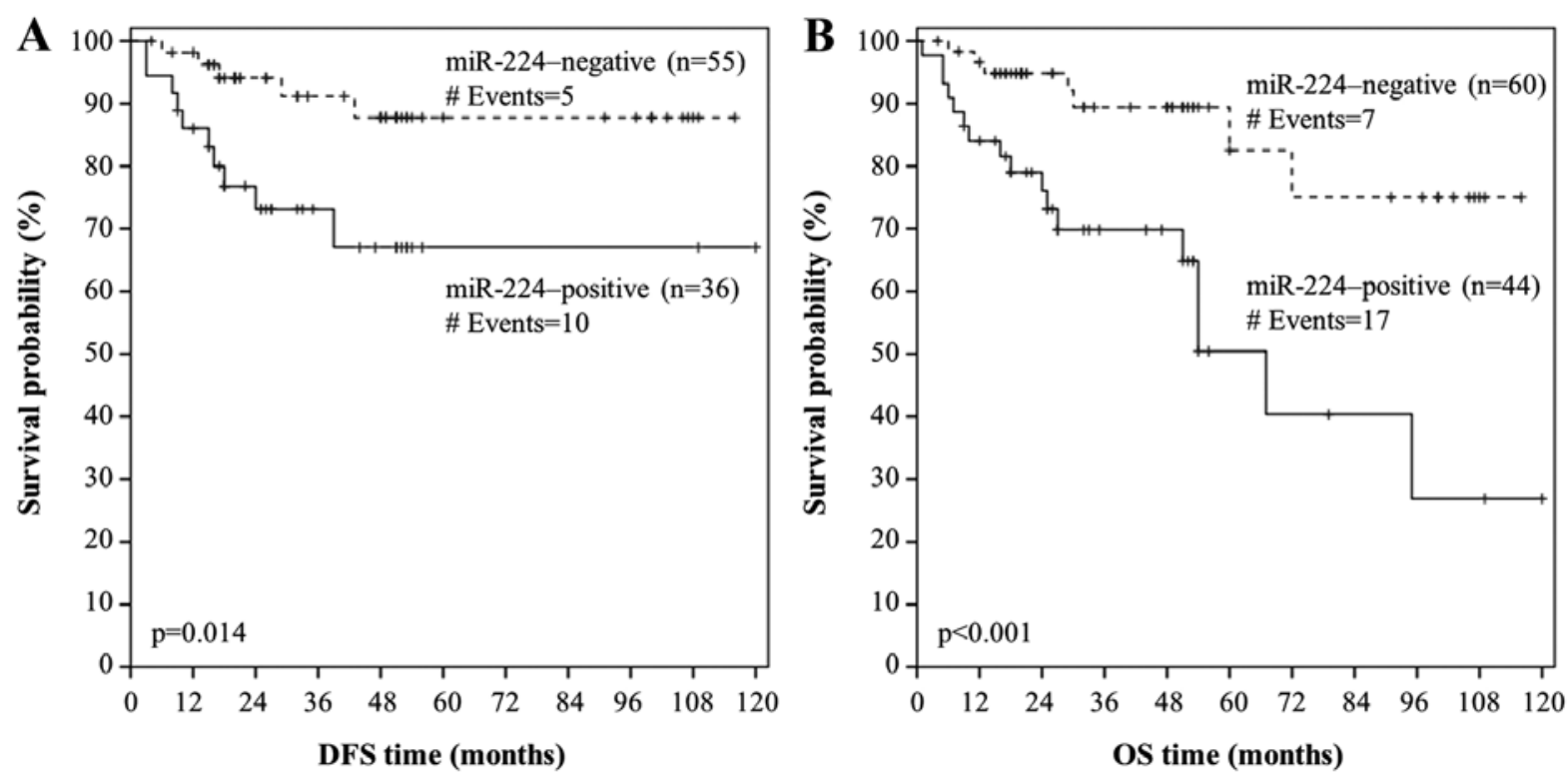

Figure 4. Kaplan-Meier survival curves for (A) DFS and (B) OS of colorectal adenocarcinoma patients. miR-224 expression was shown to have an unfavorable prognostic value in this malignancy, as patients with miR-224-positive colorectal adenocarcinoma have significantly shorter DFS and OS intervals, compared to those with miR-224-negative tumors.

that patients with miR-224-positive colorectal adenocarcinoma were more likely to succumb to their disease later than patients with a miR-224-negative malignancy $(\mathrm{p}<0.001$; Fig. 4B).

In the multivariate Cox regression analysis (Table IV), miR-224 positivity remained a statistically significant indicator of poor OS in colorectal adenocarcinoma, independent of tumor size, histological grade, invasion, nodal status, and presence of distant metastases $(\mathrm{HR}=4.41,95 \% \mathrm{CI}=1.72-11.34$, $\mathrm{p}=0.002$ ). More importantly, miR-224 expression retained its independent prognostic significance in colorectal adenocarcinoma $(\mathrm{HR}=3.74,95 \% \mathrm{CI}=1.48-9.44, \mathrm{p}=0.005)$ even when the multivariate Cox regression model was adjusted for tumor size, histological grade and TNM stage.
Prognostic value of miR-224 expression in colorectal adenocarcinoma patients, stratified according to tumor histological grade, nodal status and disease stage. Owing to the fact that patients with well-differentiated tumors as well as those without regional nodal metastases or, at least, without distant metastases are substantially different from patients with poorly-differentiated colorectal adenocarcinoma or advanced-stage patients with metastases, respectively, in terms of their prognosis and postoperative treatment, Kaplan-Meier survival analysis was carried out to assess the prognostic value of miR-224 expression regarding DFS and OS for each group of patients, stratified according to the aforementioned clinicopathological features. As depicted in Figs. 5A and 6A, patients with well(grade I) or moderately-differentiated (grade II) colorectal 
Table IV. miR-224 expression and OS of colorectal adenocarcinoma patients.

OS

\begin{tabular}{lrcc}
\cline { 2 - 4 } Variable & $\mathrm{HR}^{\mathrm{b}}$ & $95 \% \mathrm{CI}^{\mathrm{c}}$ & $\mathrm{p}$-value \\
\hline Univariate analysis (n=104) & & & \\
$\quad$ miR-224 expression status & & & \\
$\quad$ Negative & 1.00 & & \\
$\quad$ Positive & 4.08 & $1.68-9.88$ & 0.002 \\
Tumor size & 1.01 & $0.99-1.03$ & 0.47 \\
Histological grade (ordinal) & 2.58 & $1.21-5.50$ & 0.014 \\
T (ordinal) & 3.76 & $1.79-7.91$ & $<0.001$ \\
Positive nodal status & 2.02 & $1.22-3.33$ & 0.006 \\
Distant metastasis & 10.90 & $4.65-25.55$ & $<0.001$ \\
TNM stage (ordinal) & 3.77 & $2.18-6.53$ & $<0.001$ \\
\hline
\end{tabular}

\begin{tabular}{llll}
$\begin{array}{l}\text { Multivariate analysis }(\mathrm{n}=104) \\
\text { miR-224 expression status }{ }^{\mathrm{a}, \mathrm{d}}\end{array}$ & & & \\
$\quad$ Negative & 1.00 & & \\
$\quad$ Positive & 4.41 & $1.72-11.34$ & 0.002 \\
Tumor size & 0.97 & $0.94-1.01$ & 0.13 \\
Histological grade (ordinal) & 2.60 & $0.97-6.98$ & 0.057 \\
T (ordinal) & 3.12 & $1.33-7.35$ & 0.009 \\
Positive nodal status & 1.43 & $0.77-2.66$ & 0.26 \\
Distant metastasis & 6.06 & $2.14-17.15$ & $<0.001$ \\
\hline
\end{tabular}

\begin{tabular}{llll} 
miR-224 expression status ${ }^{\mathrm{a}, \mathrm{e}}$ & & & \\
Negative & 1.00 & & \\
Positive & 3.74 & $1.48-9.44$ & 0.005 \\
Tumor size & 0.99 & $0.96-1.02$ & 0.57 \\
Histological grade (ordinal) & 1.71 & $0.74-3.98$ & 0.21 \\
TNM stage (ordinal) & 3.49 & $1.87-6.51$ & $<0.001$ \\
\hline
\end{tabular}

OS, overall survival; HR, hazard ratio; CI, confidence interval; T, tumor invasion; ${ }^{a}$ cut-off point: $37.79 \mathrm{RQU}$, equal to the 56th percentile; bestimated from Cox proportional hazard regression model; ${ }^{\mathrm{c}}$ confidence interval of the estimated HR; ${ }^{d}$ multivariate models were adjusted for tumor size, histological grade, tumor invasion $(\mathrm{T})$, patients' nodal status $(\mathrm{N})$, and presence of distant metastasis $(\mathrm{M})$; ${ }^{\mathrm{e}}$ multivariate models were adjusted for tumor size, histological grade and TNM stage.

adenocarcinoma strongly expressing miR-224 had lower DFS and OS probabilities than patients with miR-224-negative grade I/II tumors ( $\mathrm{p}=0.007$ and $\mathrm{p}<0.001$, respectively). Furthermore, node-negative (N0) patients with colorectal adenocarcinoma that was positive for miR-224 tended to relapse or succumb to their disease earlier than node-negative patients with miR-224-negative carcinomas ( $p=0.003$ in both cases), as shown by the respective Kaplan-Meier DFS and OS curves (Figs. 5B and 6B). Interestingly, miR-224 positivity predicts an unfavorable outcome also in metastasis-free patients, in terms of OS ( $\mathrm{p}=0.004$; Fig. 6C). More importantly, patients with miR-224-positive early-stage (TNM stage I/II) colorectal adenocarcinoma had lower OS rates than did patients bearing miR-224-negative early-stage tumors ( $p=0.009$; Fig. 6D).

\section{Discussion}

Colorectal adenocarcinoma, which constitutes by far the most common type of colorectal cancer, is a multistep process involving mutations in proto-oncogenes and tumor-suppressor genes with deleterious effects on the respective proteins, defective apoptosis, chromosomal or microsatellite instability, and aberrant expression of several cancer-related genes, including those being transcribed into long non-coding RNAs that are usually subjected to further splicing, resulting in the generation of tiny RNA molecules such as miRNAs $(31,32)$. These small regulatory RNAs play a key role in gene expression regulation by targeting specific complementary regions of mRNAs, mainly located in the 3'-untranslated region (3'-UTR) of the latter. Thus, miRNAs regulate post-transcriptionally the protein levels of their targets by impeding the translation of the targeted mRNAs, but can also trigger target degradation (33). Occasionally, they can also affect histone modification and DNA methylation on gene promoter sites, again resulting in modulations of expression of their targets $(34,35)$. miRNA functional studies in CRC have uncovered their implication in critical pathways such as EGFR (36), p53 (37), NFкB (38), Wnt $/ \beta$-catenin $(39,40)$ and $\beta$-catenin/APC pathways $(41)$, as well as in the regulation of EMT (38) and cancer stem cell maintenance $(38,42)$. It is therefore evident that alterations of specific miRNAs can significant contribute to colorectal carcinogenesis.

Besides having a functional role in the initiation and progression of colorectal cancer, miRNAs are about to introduce a new era of diagnostic, prognostic and therapeutic modalities for this human malignancy (43-45). These small molecules are being intensively evaluated as biomarkers and therapeutic targets for colorectal cancer (46), as their expression patterns are subjected to remarkable alterations in tumor tissues (45), blood (plasma) $(47,48)$ and feces $(49-51)$. In addition to miRNA expression signatures (48), miRNA methylation signatures may provide candidate biomarkers for the detection of malignant colonocytes, since miRNAs are epigenetically modified in CRC $(52,53)$. Polymorphisms in miRNAs or miRNA-binding sites have also particular interest, as they may modify ones risk of developing cancer $(46,54)$. Thus, miRNA transcriptome constitutes a rich pool of novel and emerging tumor biomarkers.

The oncogenic properties of miR-224 have been demonstrated by several studies using cell line models originating from different cancer types. miR-224 overexpression can affect crucial cellular processes such as apoptosis (13), cell proliferation $(13,15,55)$, cell migration and invasion $(15,55,56)$. With regard to miR-224 targets, many genes are predicted to be downregulated by this oncomiR, yet only a handful of those have been validated. The most important miR-224 targets include SMAD family member 4 (SMAD4) (55), API5 (13) and RKIP (9). In human colorectal adenocarcinoma HCT 116 cells, the common signaling transducer SMAD4 is directly downregulated by miR-224 (57), which hence attenuates the transcriptional response of TGF- $\beta$ signaling transduction to promote cell proliferation (58). Downregulation of RKIP expression is also very important in colorectal carcinogenesis, as this inhibitor of RAF1 kinase is a master modulator of many critical intracellular signaling 

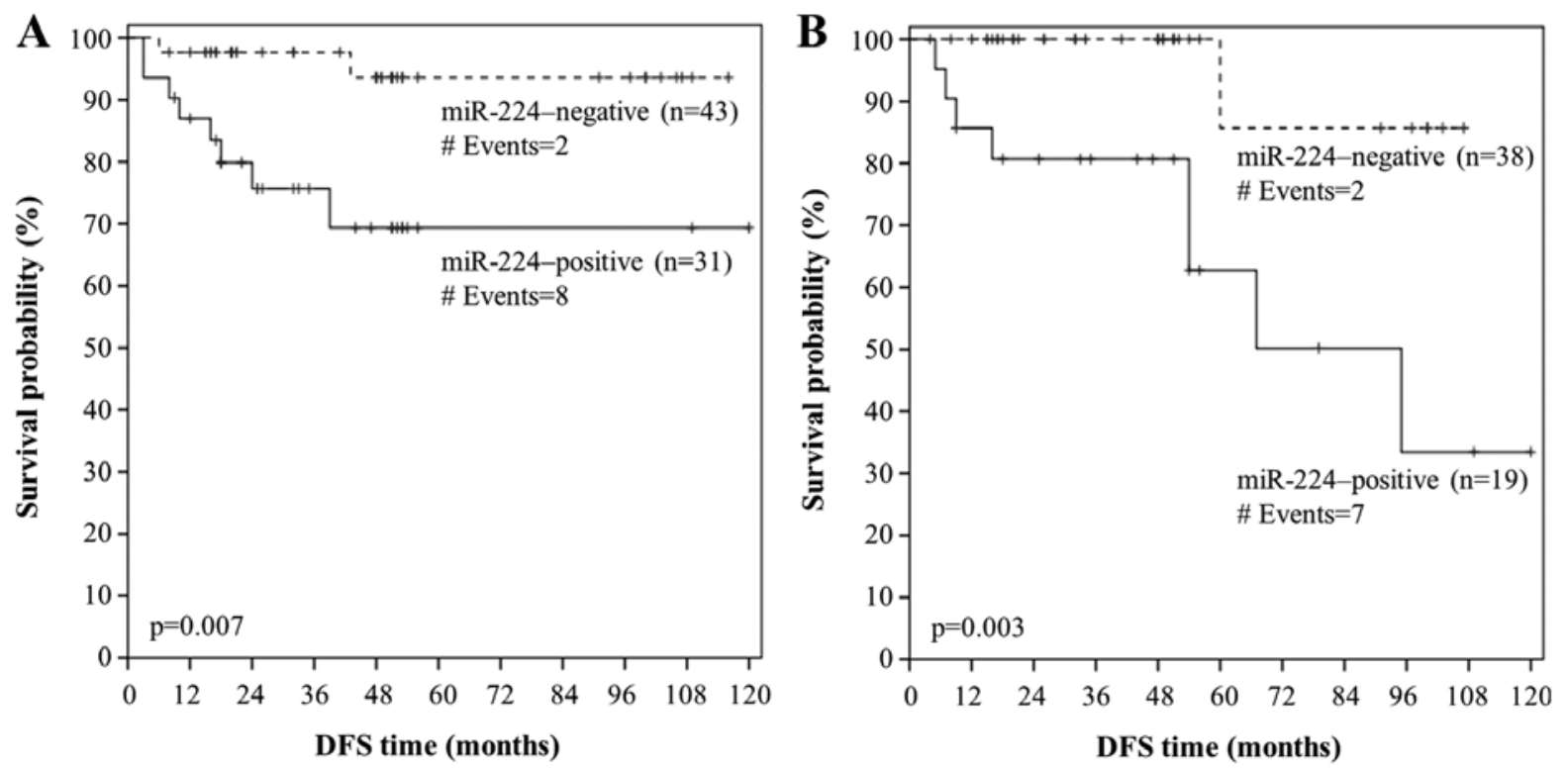

Figure 5. Kaplan-Meier survival curves for DFS of distinct groups of colorectal adenocarcinoma patients. (A) The prognosis of patients with grade I or II colorectal adenocarcinoma strongly expressing miR-224 was significantly worse than that of patients with miR-224-negative tumors of the same tumor differentiation. (B) miR-224 expression predicts poor DFS also in patients with negative nodal status.

cascades that control cellular growth, motility, apoptosis, genomic integrity and therapeutic resistance (59). Finally, as aforementioned, elimination of API5 sensitizes cancer cells to chemotherapy (22), whereas miR-224 depletion and, probably, subsequent API5 overexpression render human colorectal adenocarcinoma HT-29 cells resistant to methotrexate (60). Nonetheless, the in vivo function and the clinical significance of miR-224 overexpression in CRC remain unclear.

Our study investigated the potential diagnostic and prognostic value of miR-224 expression in colorectal adenocarcinoma. Since previous studies failed to detect circulating miR-224 (17), we used tumor tissue and adjacent non-cancerous colorectal mucosae. In accordance with previous findings (16-18), our study showed that miR-224 expression is significantly upregulated in colorectal adenocarcinoma in comparison with non-cancerous adjacent mucosae $(p<0.001)$. Taking this step further, we also showed that miR-224 expression levels can discriminate colorectal adenocarcinoma patients from normal population, as indicated by ROC analysis $(\mathrm{p}<0.001)$ and univariate binary logistic regression models $(\mathrm{p}<0.001)$. Recent studies examining the diagnostic potential of several other miRNAs, including miR-106a (61), miR-141 (62), miR-17-3p (63), miR-21 (61,62), miR-29a (64) and miR-92 (62-64), have shown that miRNAs can provide important diagnostic information with an enhanced AUC. Therefore, it would be useful to analyze miR-224 expression in combination with other miRNAs, in order to create a multiparametric panel of markers for colorectal adenocarcinoma diagnosis with relatively good accuracy. Undoubtedly, this model will need to be validated within a larger cohort and may be further improved by addition of other tumor markers.

To the best of our knowledge, this is the first study examining the prognostic significance of miR-224 expression in colorectal adenocarcinoma, in terms of DFS. Cox univariate regression analysis showed that high miR-224 expression in colorectal adenocarcinoma predicts an increased risk of relapse, and Kaplan-Meier survival analysis demonstrated significantly lower DFS rates for miR-224-positive patients. These findings seem to partially contradict the results of a previous study demonstrating that miR-224 along with miR-221* promote colorectal tumor growth and metastasis in mice (65). However, there are two major differences that should be taken into account: first, that study compared miR-224 between metastatic and non-metastatic samples, while it did not examine the prognostic potential of miR-224; second, the human material included in that study is restricted (only 20 human CRC samples) compared to our material (115 human colorectal adenocarcinoma samples).

Interestingly, the unfavorable prognostic value of miR-224 with regard to DFS is independent of established clinicopathological parameters such as histological differentiation, tumor invasion, and regional lymph node metastasis, as revealed by the multivariate Cox regression analysis. Moreover, miR-224 overexpression retained its unfavorable prognostic significance in the subgroups of colorectal adenocarcinoma patients with grade I/II tumors or negative nodal status. This is especially important when considering that about $30 \%$ of CRC patients with histopathology-negative regional lymph nodes (N0) die from metastatic disease, reflected by microscopic lymph node metastases that are overlooked by currently used techniques (66).

miR-224 expression appears to constitute also an independent unfavorable prognosticator of OS in colorectal adenocarcinoma. As demonstrated by both Kaplan-Meier and Cox regression analysis, miR-224 overexpression is an adverse prognostic factor in colorectal adenocarcinoma, as it predicts a quite 4-fold risk of death for patients, independently of the clinicopathological features of the TNM stage. This finding not only confirms the results of Liao et al, according to which 

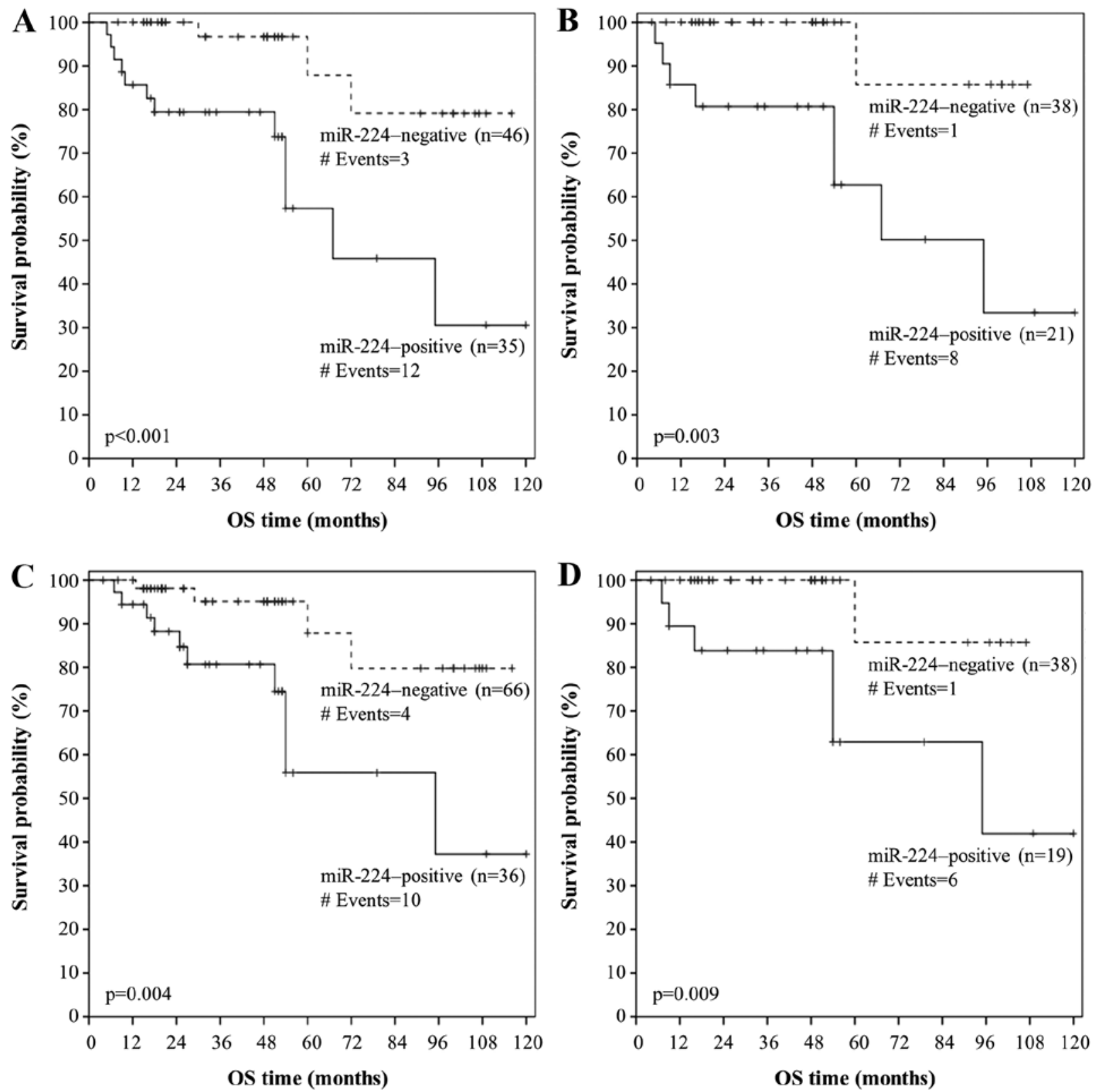

Figure 6. Kaplan-Meier survival curves for OS of distinct groups of colorectal adenocarcinoma patients. OS probabilities of patients with (A) low grade tumors, (B) negative regional lymph nodes, (C) metastasis-free disease, (D) or early TNM stage (I/II) are significantly lower when their tumors are also miR-224-negative.

miR-224 expression predicts poor outcome in CRC patients independently of tumor invasion and regional lymph node status (67), but takes it a step further by showing that miR-224 positivity, defined as expression over the optimal cut-off point (not by arbitrary choice of the median as cut-off value), is also independent of the presence (or absence) of distant metastases. In addition to the above findings, we show that high miR-224 expression constitutes a predictor of poor survival even among patients with apparently similar OS probabilities, such as those with negative regional lymph nodes, without distant metastasis, or at an early TNM stage (I or II), thus suggesting its putative future exploitation by multiparametric prognostic models composed of a gamut of molecular biomarkers with prognostic value in colorectal adenocarcinoma.
In conclusion, we demonstrated that miR-224 is upregulated in colorectal adenocarcinoma compared with adjacent non-cancerous mucosae, that miR-244 expression could be used to discriminate malignant from normal colorectal tissue, and that miR-224 positivity is an independent prognosticator of short-term relapse and poor OS in colorectal adenocarcinoma. Additional studies are hence necessitated to thoroughly evaluate the potential of miR-224 as a prognostic biomarker in colorectal adenocarcinoma.

\section{Acknowledgements}

This study was financially supported by the Commission of the European Community through the INsPiRE project (EU-FP7-REGPOT-2011-1, proposal 284460). 


\section{References}

1. Bartel DP: MicroRNAs: genomics, biogenesis, mechanism, and function. Cell 116: 281-297, 2004

2. Xu P, Vernooy SY, Guo M and Hay BA: The Drosophila microRNA Mir-14 suppresses cell death and is required for normal fat metabolism. Curr Biol 13: 790-795, 2003.

3. Brennecke J, Hipfner DR, Stark A, Russell RB and Cohen SM: bantam encodes a developmentally regulated microRNA that controls cell proliferation and regulates the proapoptotic gene hid in Drosophila. Cell 113: 25-36, 2003.

4. Weitz J, Koch M, Debus J, Hohler T, Galle PR and Buchler MW: Colorectal cancer. Lancet 365: 153-165, 2005.

5. Lynch HT and de la Chapelle A: Hereditary colorectal cancer. N Engl J Med 348: 919-932, 2003

6. Pagliuca A, Valvo C, Fabrizi E, et al: Analysis of the combined action of miR-143 and miR-145 on oncogenic pathways in colorectal cancer cells reveals a coordinate program of gene repression. Oncogene 32: 4806-4813, 2012.

7. Prueitt RL, Yi M, Hudson RS, et al: Expression of microRNAs and protein-coding genes associated with perineural invasion in prostate cancer. Prostate 68: 1152-1164, 2008.

8. Mavridis K, Stravodimos K and Scorilas A: Downregulation and prognostic performance of microRNA 224 expression in prostate cancer. Clin Chem 59: 261-269, 2013.

9. Huang L, Dai T, Lin X, et al: MicroRNA-224 targets RKIP to control cell invasion and expression of metastasis genes in human breast cancer cells. Biochem Biophys Res Commun 425: 127-133, 2012.

10. White NM, Chow TF, Mejia-Guerrero S, et al: Three dysregulated miRNAs control kallikrein 10 expression and cell proliferation in ovarian cancer. Br J Cancer 102: 1244-1253, 2010.

11. Shen SN, Wang LF, Jia YF, Hao YQ, Zhang L and Wang H: Upregulation of microRNA-224 is associated with aggressive progression and poor prognosis in human cervical cancer. Diagn Pathol 8: 69, 2013.

12. Mees ST, Mardin WA, Sielker S, et al: Involvement of CD40 targeting miR-224 and miR-486 on the progression of pancreatic ductal adenocarcinomas. Ann Surg Oncol 16: 2339-2350, 2009.

13. Wang Y, Lee AT, Ma JZ, et al: Profiling microRNA expression in hepatocellular carcinoma reveals microRNA-224 up-regulation and apoptosis inhibitor-5 as a microRNA-224-specific target. J Biol Chem 283: 13205-13215, 2008

14. Ladeiro Y, Couchy G, Balabaud C, et al: MicroRNA profiling in hepatocellular tumors is associated with clinical features and oncogene/tumor suppressor gene mutations. Hepatology 47: 1955-1963, 2008.

15. Li Q, Wang G, Shan JL, et al: MicroRNA-224 is upregulated in HepG2 cells and involved in cellular migration and invasion. J Gastroenterol Hepatol 25: 164-171, 2010.

16. Arndt GM, Dossey L, Cullen LM, et al: Characterization of global microRNA expression reveals oncogenic potential of miR-145 in metastatic colorectal cancer. BMC Cancer 9: 374, 2009.

17. Vega AB, Pericay C, Moya I, et al: microRNA expression profile in stage III colorectal cancer: Circulating miR-18a and miR-29a as promising biomarkers. Oncol Rep 30: 320-326, 2013.

18. Wang YX, Zhang XY, Zhang BF, Yang CQ, Chen XM and Gao HJ: Initial study of microRNA expression profiles of colonic cancer without lymph node metastasis. J Dig Dis 11: 50-54, 2010.

19. Nikiforova MN, Tseng GC, Steward D, Diorio D and Nikiforov YE: MicroRNA expression profiling of thyroid tumors: biological significance and diagnostic utility. J Clin Endocrinol Metab 93: 1600-1608, 2008.

20. Lu S, Wang S, Geng S, Ma S, Liang Z and Jiao B: Upregulation of microRNA-224 confers a poor prognosis in glioma patients. Clin Transl Oncol 15: 569-574, 2013.

21. Li Z, Lu J, Sun M, et al: Distinct microRNA expression profiles in acute myeloid leukemia with common translocations. Proc Natl Acad Sci USA 105: 15535-15540, 2008.

22. Rigou P, Piddubnyak V, Faye A, et al: The antiapoptotic protein AAC-11 interacts with and regulates Acinus-mediated DNA fragmentation. EMBO J 28: 1576-1588, 2009.

23. Koci L, Chlebova K, Hyzdalova M, et al: Apoptosis inhibitor 5 (API-5; AAC-11; FIF) is upregulated in human carcinomas in vivo. Oncol Lett 3: 913-916, 2012.
24. Koelzer VH, Karamitopoulou E, Dawson H, Kondi-Pafiti A Zlobec I and Lugli A: Geographic analysis of RKIP expression and its clinical relevance in colorectal cancer. Br J Cancer 108: 2088-2096, 2013

25. Chatzigeorgiou A, Lyberi M, Chatzilymperis G, Nezos A and Kamper E: CD40/CD40L signaling and its implication in health and disease. Biofactors 35: 474-483, 2009.

26. Georgopoulos NT, Merrick A, Scott N, Selby PJ, Melcher A and Trejdosiewicz LK: CD40-mediated death and cytokine secretion in colorectal cancer: a potential target for inflammatory tumour cell killing. Int J Cancer 121: 1373-1381, 2007.

27. Nagtegaal ID, Quirke P and Schmoll HJ: Has the new TNM classification for colorectal cancer improved care? Nature reviews. Clin Oncol 9: 119-123, 2012

28. Schmittgen TD and Livak KJ: Analyzing real-time PCR data by the comparative C(T) method. Nat Protoc 3: 1101-1108, 2008.

29. Livak KJ and Schmittgen TD: Analysis of relative gene expression data using real-time quantitative PCR and the 2(-Delta Delta C(T)) method. Methods 25: 402-408, 2001

30. Camp RL, Dolled-Filhart M and Rimm DL: X-tile: a new bio-informatics tool for biomarker assessment and outcomebased cut-point optimization. Clin Cancer Res 10: 7252-7259, 2004.

31. Chung DC: The genetic basis of colorectal cancer: insights into critical pathways of tumorigenesis. Gastroenterology 119: 854-865, 2000.

32. Colussi D, Brandi G, Bazzoli F and Ricciardiello L: Molecular pathways involved in colorectal cancer: implications for disease behavior and prevention. Int J Mol Sci 14: 16365-16385, 2013

33. Huntzinger E and Izaurralde $\mathrm{E}$ : Gene silencing by microRNAs: contributions of translational repression and mRNA decay. Nat Rev Genet 12: 99-110, 2011.

34. Tan Y, Zhang B, Wu T, et al: Transcriptional inhibiton of Hoxd4 expression by miRNA-10a in human breast cancer cells. BMC Mol Biol 10: 12, 2009.

35. Hawkins PG and Morris KV: RNA and transcriptional modulation of gene expression. Cell Cycle 7: 602-607, 2008.

36. Mlcochova J, Faltejskova P, Nemecek R, Svoboda M and Slaby O: MicroRNAs targeting EGFR signalling pathway in colorectal cancer. J Cancer Res Clin Oncol 139: 1615-1624, 2013.

37. Ma Q, Wang X, Li Z, et al: microRNA-16 represses colorectal cancer cell growth in vitro by regulating the p53/survivin signaling pathway. Oncol Rep 29: 1652-1658,2013.

38. Ma Y, Li W and Wang H: Roles of miRNA in the initiation and development of colorectal carcinoma. Curr Pharm Des 19: 1253-1261, 2013

39. Yamada N, Noguchi S, Mori T, Naoe T, Maruo K and Akao Y: Tumor-suppressive microRNA-145 targets catenin delta-1 to regulate $\mathrm{Wnt} /$ beta-catenin signaling in human colon cancer cells. Cancer Lett 335: 332-342, 2013.

40. Fu J, Tang W, Du P, et al: Identifying microRNA-mRNA regulatory network in colorectal cancer by a combination of expression profile and bioinformatics analysis. BMC Syst Biol 6: 68,2012

41. Nagel R, le Sage C, Diosdado B, et al: Regulation of the adenomatous polyposis coli gene by the miR-135 family in colorectal cancer. Cancer Res 68: 5795-5802, 2008.

42. Yu Y, Kanwar SS, Patel BB, et al: MicroRNA-21 induces stemness by downregulating transforming growth factor beta receptor 2 (TGFbetaR2) in colon cancer cells. Carcinogenesis 33: 68-76, 2012.

43. Dong Y, Wu WK, Wu CW, Sung JJ, Yu J and Ng SS: MicroRNA dysregulation in colorectal cancer: a clinical perspective. Br J Cancer 104: 893-898, 2011.

44. Zhai $\mathrm{H}$ and $\mathrm{Ju} \mathrm{J}$ : Implications of microRNAs in colorectal cancer development, diagnosis, prognosis, and therapeutics. Front Genet 2: pii00078, 2011.

45. Menendez P, Villarejo P, Padilla D, Menendez JM and Rodriguez-Montes JA: Implications of the histological determination of microRNAs in the screening, diagnosis and prognosis of colorectal cancer. J Surg Oncol 108: 70-73, 2013.

46. Schetter AJ and Harris CC: Alterations of microRNAs contribute to colon carcinogenesis. Semin Oncol 38: 734-742, 2011.

47. Madhavan D, Cuk K, Burwinkel B and Yang R: Cancer diagnosis and prognosis decoded by blood-based circulating microRNA signatures. Front Genet 4: 116, 2013. 
48. Bartley AN, Yao H, Barkoh BA, et al: Complex patterns of altered MicroRNA expression during the adenoma-adenocarcinoma sequence for microsatellite-stable colorectal cancer. Clin Cancer Res 17: 7283-7293, 2011.

49. Kalimutho M, Del Vecchio Blanco G, Di Cecilia S, et al: Differential expression of miR-144* as a novel fecal-based diagnostic marker for colorectal cancer. J Gastroenterol 46 1391-1402, 2011.

50. Xiao YF, Yong X, Fan YH, Lu MH, Yang SM and Hu CJ: microRNA detection in feces, sputum, pleural effusion and urine: Novel tools for cancer screening (Review). Oncol Rep 30: 535-544, 2013.

51. Wu CW, Ng SS, Dong YJ, et al: Detection of miR-92a and miR-21 in stool samples as potential screening biomarkers for colorectal cancer and polyps. Gut 61: 739-745, 2012.

52. Cho WC: Epigenetic alteration of microRNAs in feces of colorectal cancer and its clinical significance. Expert Rev Mol Diagn 11: 691-694, 2011.

53. Kalimutho M, Di Cecilia S, Del Vecchio Blanco G, et al: Epigenetically silenced $\mathrm{miR}-34 \mathrm{~b} / \mathrm{c}$ as a novel faecal-based screening marker for colorectal cancer. Br J Cancer 104: 1770-1778, 2011.

54. Srivastava K and Srivastava A: Comprehensive review of genetic association studies and meta-analyses on miRNA polymorphisms and cancer risk. PLoS One 7: e50966, 2012.

55. Yao G, Yin M, Lian J, et al: MicroRNA-224 is involved in transforming growth factor-beta-mediated mouse granulosa cell proliferation and granulosa cell function by targeting Smad4. Mol Endocrinol 24: 540-551, 2010.

56. Li X, Shen Y, Ichikawa H, Antes T and Goldberg GS: Regulation of miRNA expression by Src and contact normalization: effects on nonanchored cell growth and migration. Oncogene 28 4272-4283, 2009.

57. Wang Y, Ren J, Gao Y, et al: MicroRNA-224 targets SMAD family member 4 to promote cell proliferation and negatively influence patient survival. PLoS One 8: e68744, 2013.
58. Miyaki M and Kuroki T: Role of Smad4 (DPC4) inactivation in human cancer. Biochem Biophys Res Commun 306: 799-804, 2003.

59. Al-Mulla F, Bitar MS, Taqi Z and Yeung KC: RKIP: much more than Raf kinase inhibitory protein. J Cell Physiol 228: 1688-1702, 2013

60. Mencia N, Selga E, Noe V and Ciudad CJ: Underexpression of miR-224 in methotrexate resistant human colon cancer cells. Biochem Pharmacol 82: 1572-1582, 2011.

61. Link A, Balaguer F, Shen Y, et al: Fecal MicroRNAs as novel biomarkers for colon cancer screening. Cancer Epidemiol Biomarkers Prev 19: 1766-1774, 2010.

62. Cheng H, Zhang L, Cogdell DE, et al: Circulating plasma MiR-141 is a novel biomarker for metastatic colon cancer and predicts poor prognosis. PLoS One 6: e17745, 2011.

63. Ng EK, Chong WW, Jin H, et al: Differential expression of microRNAs in plasma of patients with colorectal cancer: a potential marker for colorectal cancer screening. Gut 58: 1375-1381, 2009

64. Huang Z, Huang D, Ni S, Peng Z, Sheng W and Du X: Plasma microRNAs are promising novel biomarkers for early detection of colorectal cancer. Int J Cancer 127: 118-126, 2010.

65. Yuan K, Xie K, Fox J, et al: Decreased levels of miR-224 and the passenger strand of miR-221 increase MBD2, suppressing maspin and promoting colorectal tumor growth and metastasis in mice. Gastroenterology 145: 853-864, 2013.

66. Hyslop T and Waldman SA: Molecular staging of node negative patients with colorectal cancer. J Cancer 4: 193-199, 2013.

67. Liao WT, Li TT, Wang ZG, et al: microRNA-224 promotes cell proliferation and tumor growth in human colorectal cancer by repressing PHLPP1 and PHLPP2. Clin Cancer Res 19: 4662-4672, 2013. 